\title{
Inhibition of EZH2 ameliorates bacteria-induced liver injury by repressing RUNX1 in dendritic cells
}

Yanan Wang ${ }^{1}$, Qiwei Wang ${ }^{1}$, Bei Wang ${ }^{2}$, Yuting Gu${ }^{3}$, Hongshuang Yu², Wanlin Yang ${ }^{4}$, Xiaohui Ren², Fengtao Qian², Xiaonan Zhao ${ }^{4}$, Yichuan Xiao $\mathbb{B}^{2}$, Yanyun Zhang ${ }^{1,2,4}$, Min Jin ${ }^{2,3}$ and Meiling Zhu ${ }^{5}$

\begin{abstract}
Fulminant hepatic failure (FHF) is a clinical syndrome characterized by a sudden and severe impairment in liver function. However, the precise mechanism of immune dysregulation that is significant to FHF pathogenesis remains unclear. Enhancer of zeste homolog 2 (EZH2) has been implicated in inflammation as a regulator of immune cell function. In this study, we investigated the role of EZH2 in an animal model of human FHF induced by Propionibacterium acnes (P. acnes) and lipopolysaccharide (LPS). We demonstrated that EZH2 depletion in dendritic cells (DCs) and pharmacological inhibition of EZH2 using GSK126 both significantly ameliorated liver injury and improved the survival rates of mice with $P$. acnes plus LPS-induced FHF, which could be attributed to the decreased infiltration and activation of $\mathrm{CD} 4^{+} \mathrm{T}$ cells in the liver, inhibition of T helper 1 cells and induction of regulatory $T$ cells. The expression of EZH2 in DCs was increased after $P$. acnes administration, and EZH2 deficiency in DCs suppressed DC maturation and prevented DCs from efficiently stimulating $C D 4^{+}$T-cell proliferation. Further mechanistic analyses indicated that EZH2 deficiency directly increased the expression of the transcription factor RUNX1 and thereby suppressed the immune functions of DCs. The functional dependence of EZH2 on RUNX1 was further illustrated in DC-specific Ezh2-deficient mice. Taken together, our findings establish that EZH2 exhibits anti-inflammatory effects through inhibition of RUNX1 to regulate DC functions and that inhibition of EZH2 alleviates $P$. acnes plus LPS-induced FHF, probably by inhibiting DC-induced adaptive immune responses. These results highlight the effect of EZH2 on DCs, serving as a guide for the development of a promising immunotherapeutic strategy for FHF.
\end{abstract}

\section{Introduction}

Fulminant hepatic failure (FHF), also known as acute liver failure, is characterized by a sudden and severe impairment of liver function, which develops secondary to infection, toxin exposure, or immune-mediated attack and is a rare but potentially fatal disease $\mathrm{e}^{1-3}$. The global mortality rate is very high; however, there are no definitive

\footnotetext{
Correspondence: Yanyun Zhang (yyzhang@sibs.ac.cn) or Min Jin (mjin@sibs.ac.cn) or Meiling Zhu (zhumeiling@xinhuamed.com.cn) 'Shanghai Institute of Immunology, Shanghai Jiao Tong University School of Medicine, Shanghai, China

${ }^{2}$ CAS Key Laboratory of Tissue Microenvironment and Tumor, Shanghai Institute of Nutrition and Health, Chinese Academy of Sciences, Shanghai, China

Full list of author information is available at the end of the article These authors contributed equally: Yanan Wang, Qiwei Wang Edited by H.-U. Simon
}

therapeutic strategies for FHF other than liver transplantation, and the mechanisms leading to this dramatic and challenging syndrome remain to be elucidated. Inflammatory responses are involved in the pathophysiology of hepatic cell death and liver injury, and they are also associated with hepatic regeneration failure. Hence, suppression of hepatic inflammation and excess tissue injury may represent a potential approach to limit the pathology of FHF.

Mice injected with heat-killed Propionibacterium acnes (P. acnes) followed by lipopolysaccharide (LPS) are one of the most commonly used animal models of fulminant hepatitis $^{4-7}$. This model can be classified into two different phases: the priming phase, in which the injection of $P$. acnes generates granulomas, and the elicitation phase, in which LPS activates granuloma-forming cells, leading

\section{(c) The Author(s) 2020}

(c) (i) Open Access This article is licensed under a Creative Commons Attribution 4.0 International License, which permits use, sharing, adaptation, distribution and reproduction cc) in any medium or format, as long as you give appropriate credit to the original author(s) and the source, provide a link to the Creative Commons license, and indicate if changes were made. The images or other third party material in this article are included in the article's Creative Commons license, unless indicated otherwise in a credit line to the material. If material is not included in the article's Creative Commons license and your intended use is not permitted by statutory regulation or exceeds the permitted use, you will need to obtain permission directly from the copyright holder. To view a copy of this license, visit http://creativecommons.org/licenses/by/4.0/. 
to severe liver injury. Dendritic cells (DCs), as antigenrepresenting cells, play a key role in the initiation of immune responses ${ }^{8}$. They have been suggested to play an active role in the development of liver failure, and the distribution and number of DCs reflect the progression of liver failure ${ }^{9}$. Moreover, DCs have been implicated in the control of acute liver injury ${ }^{10-12}$. Our previous data indicate that $P$. acnes-induced granuloma formation involves a DC-induced adaptive response ${ }^{13}$. We and others have demonstrated the critical roles of DCs in liver injury $^{14-16}$, and we previously clarified that the DCinduced adaptive response participates in $P$. acnes plus LPS-induced liver injury ${ }^{13,14}$. The recruitment of DCs to the liver is a prerequisite for liver injury in this model. In the priming phase of $P$. acnes plus LPS-induced FHF, DCs and $\mathrm{T}$ cells cluster in the liver, leading to further proliferation and polarization of $\mathrm{CD} 4^{+} \mathrm{T}$ cells. DC precursors are recruited into the circulation by $P$. acnes administration, migrate into the perisinusoidal space and differentiate into mature DCs migrating to the portal area to interact with $\mathrm{T}$ cells and initiate $\mathrm{T}$ cell-mediated immune responses ${ }^{13,17}$. Therefore, inhibition of inflammatory infiltration and immune responses initiated by DCs would be beneficial for the treatment of FHF.

Epigenetic mechanisms are known to play crucial roles in the development and functions of DCs ${ }^{18,19}$. Enhancer of zeste homolog 2 (EZH2) is a core catalytic subunit of polycomb repressive complex 2 (PRC2), which mediates gene silencing by catalyzing the trimethylation of lysine 27 on histone $\mathrm{H} 3$ (H3K27me3) within the gene promoter region $^{20,21}$. EZH2 has been identified to play essential roles in immune regulation and many diseases. EZH2 has been shown to control B cell development and function $^{22,23}$ and regulate the differentiation of $\mathrm{CD} 4^{+} \mathrm{T}$ cells into both Th1 and Th2 cells, as well as $\mathrm{CD}^{+}{ }^{+} \mathrm{T}$-cell plasticity $^{24}$. We and others previously reported the pivotal roles of EZH2 in regulating the T-cell response and emphasized this protein as a novel target for immunotherapy ${ }^{25,26}$. EZH2 was identified as an important regulator of macrophage activation and autoimmune inflammation ${ }^{27}$. EZH2 is also involved in many liver diseases and contributes to pathogenesis ${ }^{28,29}$. Whether targeting EZH2 is an effective treatment for FHF and the underlying mechanisms remain elusive.

EZH2 regulates the integrin signaling and adhesion dynamics of neutrophils and $\mathrm{DCs}^{30}$, and the majority of cases of histiocytic and dendritic cell neoplasms show strong EZH2 expression ${ }^{31}$. More recently, it was shown that EZH2 plays a role in regulating the activation of DCs participating in the epigenetic mechanism of allergen immunotherapy $^{32}$. Therefore, we hypothesized that the role of EZH2 in controlling DC functions may have a profound effect on the treatment of disease. In this study, using the $P$. acnes plus LPS-induced FHF model, we uncovered that deficiency in EZH2 or inhibition of EZH2 histone methyltransferase activity significantly ameliorated liver injury and improved the survival rates of mice subjected to $P$. acnes plus LPS-induced FHF, which could be attributed to decreased infiltration and activation of $\mathrm{CD}^{+} \mathrm{T}$ cells in the liver, inhibition of $\mathrm{T}$ helper 1 cells and induction of regulatory $\mathrm{T}$ cells. We also provide molecular evidence that EZH2 suppresses the transcription factor RUNX1 to regulate DC maturation and liver injury.

\section{Materials and methods \\ Mice}

C57BL/6 mice were purchased from the Shanghai Laboratory Animal Center of the Chinese Academy of Sciences (Shanghai, China). Ezh2-floxed mice ${ }^{27}$ were crossed with $\mathrm{Cd11c}$-Cre mice originally obtained from The Jackson Laboratory (Bar Harbor, ME, USA) to generate offspring with Ezh2-deficient DCs $\left(E z h 2^{\mathrm{f} / \mathrm{f}} C d 11 c\right.$ Cre; termed $E z h 2^{\mathrm{D}-1-}$ ). All mice were maintained in a specific pathogen-free facility, and all animal experiments were conducted in accordance with protocols approved by the institutional Biomedical Research Ethics Committee, Shanghai Institutes for Biological Sciences, Chinese Academy of Sciences.

\section{Induction of liver injury}

Female wild-type (WT) and $E z h 2^{\mathrm{D}-1-} \mathrm{C} 57 \mathrm{BL} / 6$ mice (8-10 weeks old) were injected intravenously with $1 \mathrm{mg}$ of heat-killed P. acnes suspended in $100 \mu \mathrm{L}$ of phosphatebuffered saline (PBS). For survival analysis, mice were injected intravenously with $1 \mu \mathrm{g}$ of LPS in $100 \mu \mathrm{L}$ of PBS on day 7 after $P$. acnes priming. For the indicated experiments, $50 \mathrm{mg} / \mathrm{kg}$ Ro 5-3335 (Target Mol, Wellesley Hills, MA, USA), $25 \mathrm{mg} / \mathrm{kg}$ GSK126 (Selleckchem, Houston, TX, USA) or DMSO (Sigma-Aldrich, St. Louis, MO, USA) was injected intraperitoneally on days 0,2 , 4 , and 6.

\section{Histological analysis}

Liver specimens were fixed in 4\% PFA and embedded in paraffin. Deparaffinized sections $(5-10 \mu \mathrm{m})$ were stained with hematoxylin and eosin. Semiquantitative analysis of the status of liver inflammation was performed in a blinded manner as previously described ${ }^{33,34}$. Briefly, the H\&E-stained liver slides were scored by a pathologist in a "blinded fashion" to determine the degree of inflammation as follows: $0=$ no infiltration, $1=\mathrm{minimal} / \mathrm{slight}$ infiltration, $2=$ moderate infiltration, and $3=$ severe infiltration.

\section{Cytokine analysis}

The levels of IFN- $\gamma$, IL-5, IL-17, TNF- $\alpha$, and IL- 6 in the serum were assessed with enzyme-linked immunosorbent assay kits (R\&D Systems, Minneapolis, MN, USA) according to the manufacturer's instructions. 


\section{Isolation of mononuclear cells (MNCs)}

Spleen and liver samples from mice were minced and filtered through nylon mesh (BD Falcon, Franklin Lakes, NJ, USA) to obtain a cell suspension. Spleen and liver MNCs were obtained from the cell suspensions by Ficoll (Fresenius Kabi Norge AS, Norway) and Percoll (GE Healthcare, Boston, MA, USA) density gradient centrifugation protocols, which were performed separately according to the manufacturer's instructions.

\section{Isolation and generation of mouse DCs}

To isolate liver DCs, CD11c ${ }^{+}$cells were positively sorted from liver MNCs using MACS CD11c microbeads (Miltenyi Biotec, Bergisch Gladbach, Germany). For the generation of mouse DCs, mouse bone marrow cells were obtained by flushing the humerus and femur bones with PBS containing 2\% FBS (Gibco, Grand Island, NY, USA). DCs were cultured in the presence of granulocyte-macrophage colony-stimulating factor (GM-CSF; $10 \mathrm{ng} / \mathrm{mL}$ ) and interleukin (IL)-4 (5 ng/mL) (both from PeproTech, Rocky Hill, NJ, USA) for 5 days to induce immature DCs (immature DC induction phase). The cells were further incubated with GM-CSF and tumor necrosis factor (TNF)- $\alpha(50 \mathrm{ng} / \mathrm{mL}$; PeproTech) on type I collagen-coated plates for 3 more days to induce mature DCs (mature DC induction phase). LPS $(0.1 \mu \mathrm{g} / \mathrm{mL})$ was added on day 7 to further promote DC maturation.

\section{Mixed lymphocyte reaction (MLR)}

Generated DCs were lethally irradiated (30 Gy). Then, the DCs were cultured in graded doses with $\mathrm{CD} 4^{+} \mathrm{T}$ cells $\left(3 \times 10^{5}\right.$ cells/well) isolated from naive $\mathrm{BALB} / \mathrm{c}$ mice in RPMI 1640 medium for 5 days. $\left[{ }^{3} \mathrm{H}\right]$ Thymidine $(1 \mu \mathrm{Ci} /$ well, Shanghai Institute of Applied Physics, Chinese Academy of Sciences, China) was added $18 \mathrm{~h}$ before the end of the culture period. The cells were then harvested onto glass fiber mats for measurement of $\left[{ }^{3} \mathrm{H}\right]$ thymidine incorporation.

\section{Flow cytometric analysis}

Cells were stained with the following antibodies obtained from BD Biosciences (Franklin Lakes, NJ, USA), eBioscience, or BioLegend (San Diego, CA, USA): antiCD4 (BioLegend, 100451), anti-CXCR3 (eBioscience, 12-1831-82), anti-CCR7 (eBioscience, 12-1971-82), antiCD44 (eBioscience, 12-0441-83), anti-CD62L (BioLegend, 104406), anti-CD11c (BD Bioscience, 553801), antiMHCII (BioLegend, 107631), anti-CD80 (BD Biosciences, 553769), anti-CD86 (BD Biosciences, 553692), anti-B220 (eBioscience, 12-0452-83), anti-CD8 (BD Bioscience, 553032), anti-CD103 (BD Bioscience, 557495), anti-CD45 (BioLegend, 103132), and anti-CD11b (BioLegend, 101263). For Th1 and Treg analyses, cells were stained for surface markers, permeabilized with the Intracellular Fixation and Permeabilization Buffer Set (eBioscience, 88-8824-00), and then stained with antiIFN- $\gamma$ (BioLegend, 505825) and anti-FOXP3 (eBioscience, 17-5773-82) antibodies. The following antibodies were obtained from Cell Signaling Technology (Danvers, Massachusetts, USA): anti-Ezh2 (\#5246), anti-Runx1 (\#4336), and anti-H3K27me3 (\#9733). The Alexa Fluor ${ }^{\mathrm{TM}}$ 488 Goat Anti-Rabbit SFX Kit from Invitrogen (Carlsbad, CA, USA) was used as a secondary antibody. Multicolor flow cytometric analysis was performed using a CytoFLEX LX (Beckman Coulter, Indianapolis, IN, USA).

\section{Quantitative real-time PCR and a chromatin immunoprecipitation (ChIP) assay}

Total RNA was extracted from tissues or cells at the indicated time points and subsequently reverse transcribed using the Reverse Transcription System (Takara, Shiga, Japan). Quantitative real-time PCR was performed using SYBR Green PCR mix on the ViiA 7 RealTime PCR System (Applied Biosystems, Foster City, CA, USA). The reaction protocol used was $95^{\circ} \mathrm{C}$ for $5 \mathrm{~min}$, followed by 35 cycles of $95^{\circ} \mathrm{C}$ for $15 \mathrm{~s}, 60^{\circ} \mathrm{C}$ for $60 \mathrm{~s}$, and $72{ }^{\circ} \mathrm{C}$ for $5 \mathrm{~min}$. $\beta$-actin was used as an internal control to normalize differences in the amount of total RNA among the samples. ChIP was performed according to the manufacturer's recommendations (Millipore, Billerica, MA, USA). The primers are listed in Supplementary Table 1.

\section{Statistical analysis}

All values are presented as the mean \pm S.E.M. Significant differences were evaluated using an independent-sample $t$ test or the Wilcoxon rank test, and multiple treatment groups within individual experiments were compared by ANOVA or the Kruskal-Wallis test, followed by the Bonferroni posttest or Dunn's posttest to compare differences between groups. The log-rank test was used for survival analysis. Values of $P<0.05$ were considered significant. Sample sizes of all experiments were predetermined by calculations derived from our experience. No sample was excluded from the analyses. Animals were not randomly assigned during collection, but the strain, sex, and age of the mice were the same, and the data analysis was single masked. Investigators were not blinded to the group allocation during the experiment and outcome assessment. The number of replicates was indicated in each figure legend.

\section{Results \\ EZH2 expression increased in DCs in the liver during bacteria-induced FHF}

To determine the expression of EZH2 in FHF, P. acnes was injected intravenously into $\mathrm{C} 57 \mathrm{BL} / 6$ mice to mimic 


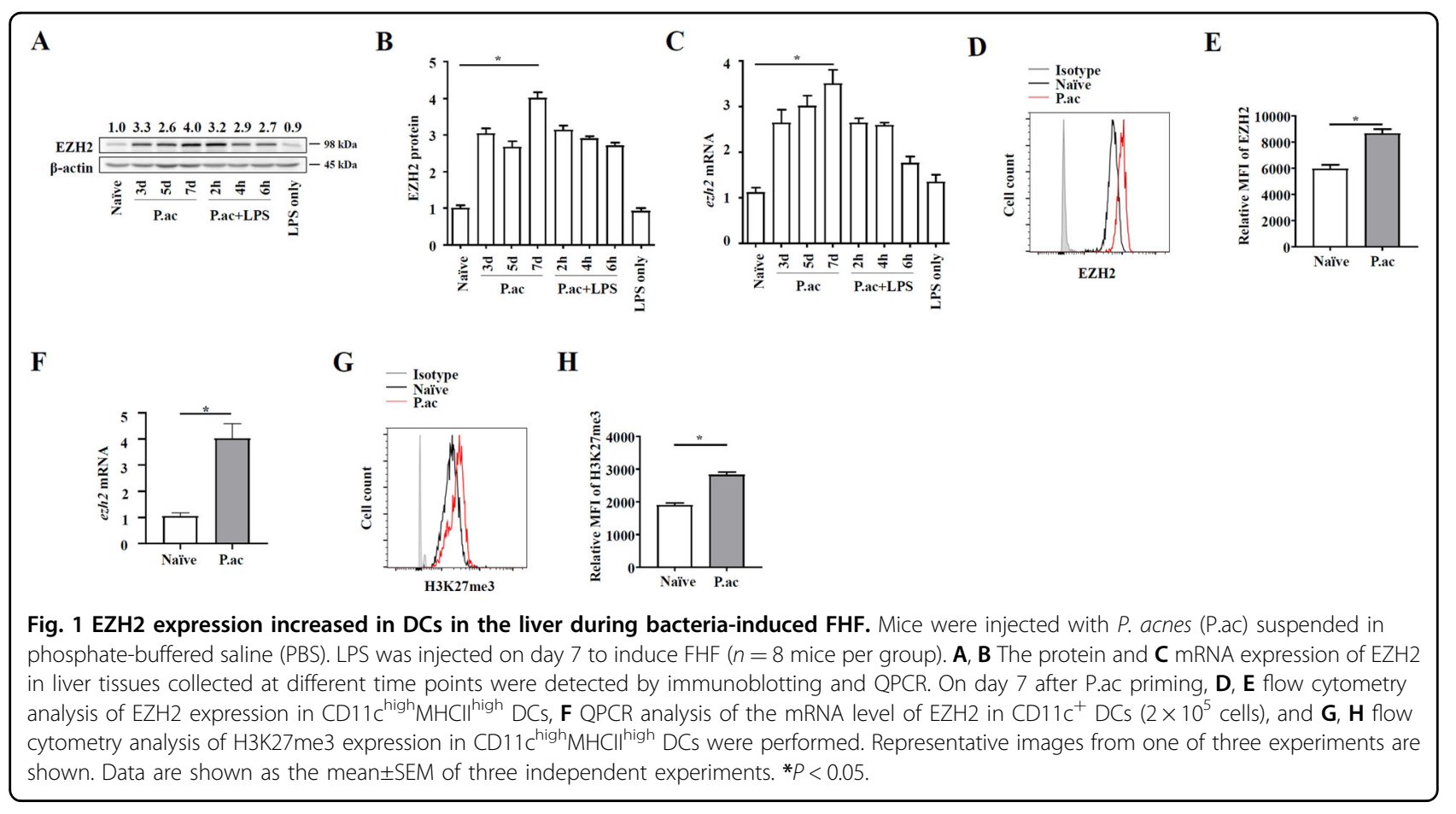

clinical FHF. The results revealed that the EZH2 protein level in the liver increased with FHF progression and reached its highest level on day 7 (Fig. 1A, B). The trend for mRNA expression was similar to that for protein expression (Fig. 1C). To explore the role of EZH2 in DCs, we detected the expression of EZH2 and H3K27me3 in CD11 $\mathrm{c}^{\text {high }} \mathrm{MHCII}^{\text {high }}$ DCs from the liver by flow cytometry. The results showed that both the protein and mRNA expression levels of EZH2 were increased in FHF DCs compared to control DCs (Supplementary Figs. 1A, 2A, and Fig. 1D-F). Consistently, the level of H3K27me3 was also increased (Fig. 1G, H). These results showed that EZH2 expression and methyltransferase activity were induced in FHF over time, suggesting that EZH2 might be related to the development of FHF.

\section{EZH2 deficiency in DCs ameliorated the severity of bacteria-induced liver injury and reduced the mortality of FHF}

To further assess the role of EZH2 in DCs in FHF, we crossed $E z h 2^{\mathrm{f} / \mathrm{f}}$ mice with mice expressing Cre recombinase from a DC-specific promoter $(C d 11 c$-Cre) to generate mice with conditional deletion of EZH2 in DCs $\left(E z h 2^{\mathrm{f} / \mathrm{f}} \mathrm{Cd} 11 \mathrm{c}\right.$-Cre, hereafter called $E z h 2^{\mathrm{D}-1-}$; Supplementary Fig. 3A). There were no differences in the generation or distribution of DCs between WT mice and $E z h 2^{\mathrm{D}-1-}$ mice $^{30}$; however, EZH2 might affect the generation of DC subpopulations in the liver and spleen during FHF (Supplementary Fig. 4A, B). To investigate the function of EZH2 in regulating DCs during FHF, $E z h 2^{\mathrm{D}-1-}$ mice and their WT littermates were challenged with $P$. acnes. All of the WT mice died within $18 \mathrm{~h}$ post LPS injection; however, the $E z h 2^{\mathrm{D}-1-}$ mice survived for more than $72 \mathrm{~h}$ (Fig. 2A). Weight loss did not change in the surviving $E z h 2^{\mathrm{D}-1-}$ mice after 7 days (Supplementary Fig. 5A). Alanine aminotransferase (ALT) and aspartate aminotransferase (AST) levels in the serum were detected, and there were dramatic decreases in the ALT and AST levels of $E z h 2^{\mathrm{D}-1-}$ mice compared with those of WT mice (Fig. 2B). Histological examination showed that large nodules, severe infiltration of lymphocytes, and granuloma formation were observed in liver tissue on day 7 post-P. acnes priming (Fig. 2C, D), and liver and spleen weights increased considerably (Fig. 2E). Quantitative real-time PCR assays revealed that the expression of inflammatory cytokine genes, such as TNF- $\alpha$, IFN- $\gamma$, and IL-6, was also elevated in livers (Fig. 2F). In contrast, $E z h 2^{\mathrm{D}-1-}$ mice displayed a normal morphology without modules, dramatically decreased infiltration of lymphocytes, reduced granuloma formation, normal liver weights, and reduced inflammatory factor levels (Fig. 2C-F). Moreover, the levels of the cytokines TNF- $\alpha$, IFN- $\gamma$, and IL- 6 in the serum were tested, and the results showed the same trend as the mRNA expression levels in the liver (Fig. 2G). These findings suggested that deletion of EZH2 in DCs effectively attenuated the severity of bacteria-induced liver injury and improved the survival rate of mice with FHF. 


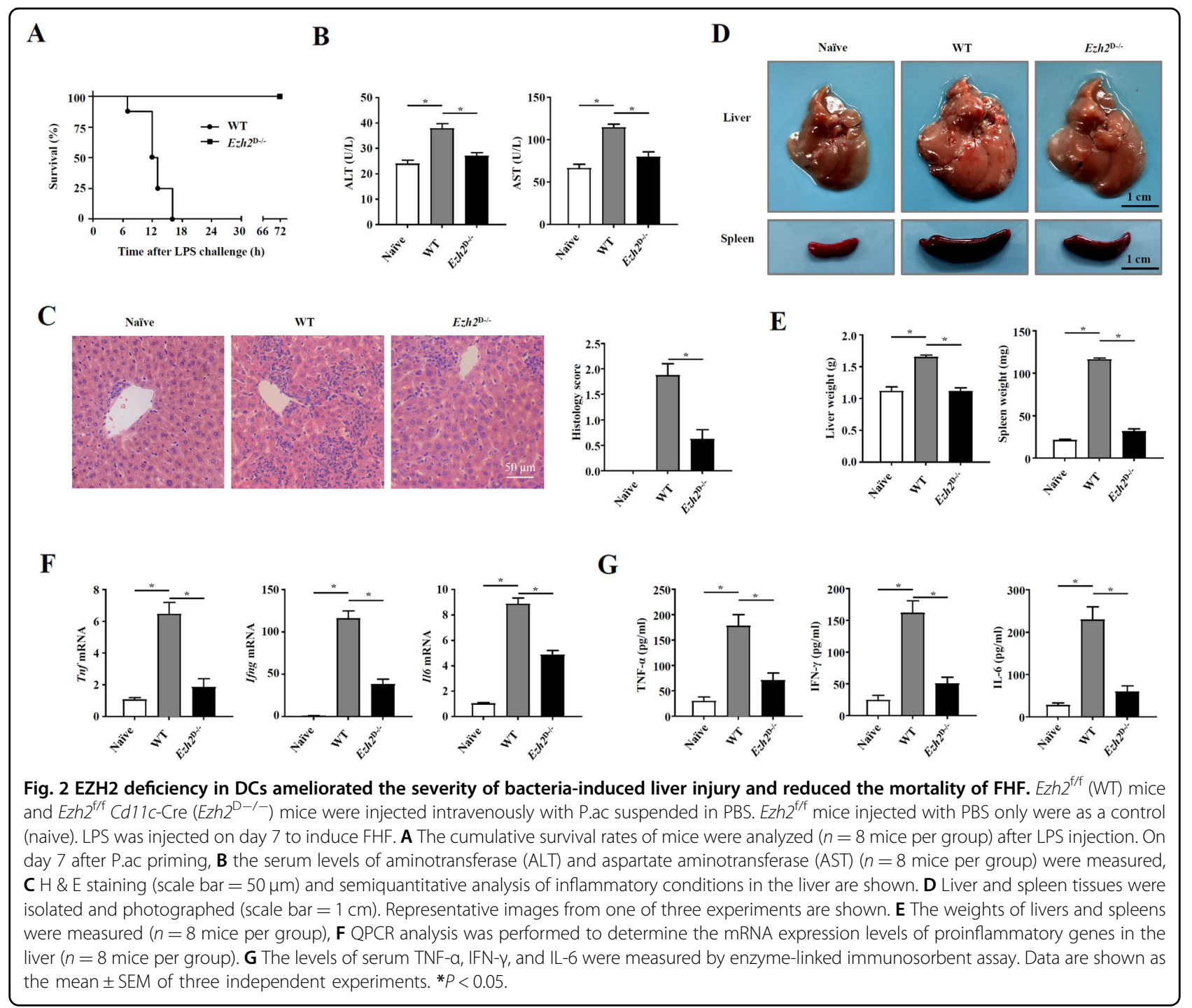

EZH2 deficiency in DCs inhibited the $\mathrm{CD}^{+}{ }^{+} \mathrm{T}$-cell response during bacteria-induced liver injury

DCs elicit an inflammatory response as antigenpresenting cells by presenting antigens to $\mathrm{CD} 4^{+} \mathrm{T}$ cells. We analyzed the numbers of mononuclear cells (MNCs) and $\mathrm{CD}^{+}{ }^{+} \mathrm{T}$ cells to determine whether EZH2 deficiency affects the infiltration of $\mathrm{CD}^{+}{ }^{+} \mathrm{T}$ cells in liver injury. The results showed that the numbers of MNCs in the liver and spleen were both decreased in $E z h 2^{\mathrm{D}-1-}$ mice and that the percentage of $\mathrm{CD}^{+}{ }^{+} \mathrm{T}$ cells in the MNC population was decreased, with the same result found in the spleen (Fig. 3A, B). Then, we analyzed the function of CD4 ${ }^{+}$ $\mathrm{T}$ cells in the liver of $E z h 2^{\mathrm{D}-1-}$ and WT mice challenged with $P$. acnes. The expression of the chemokines CXCR3 and CCR7 in the liver of $E z h 2^{\mathrm{D}-1-}$ mice was considerably reduced, suggesting that the deletion of EZH2 suppressed the chemotaxis of pathogenic $\mathrm{CD}^{+}{ }^{+} \mathrm{T}$ cells into the liver (Fig. 3C). In addition, CD $4^{+}$T-cell activation was suppressed in $E z h 2^{\mathrm{D}-1-}$ mice, as indicated by decreased expression of CD44 and increased expression of CD62L (Fig. 3D). We previously identified Th1 cells as central players in the pathogenesis of $P$. acnes-induced liver injury ${ }^{4}$. Thus, serum levels of the Th1 cytokine IFN- $\gamma$, Th2 cytokine IL-5, and additional proinflammatory cytokine IL-17 were determined. The results showed that the deletion of EZH2 significantly reduced the levels of IFN- $\gamma$ and IL-17 but had no effect on IL-5 production (Fig. 3E). Intracellular staining for IFN- $\gamma$ further confirmed the reduction in the IFN- $\gamma$-positive cell percentage within $\mathrm{CD}^{+} \mathrm{T}$ cells (Fig. 3F). Moreover, deletion of EZH2 increased the percentage of $\mathrm{CD}^{+}{ }^{+}$Foxp $3^{+}$Tregs (Fig. 3G), although the absolute number of Tregs was not increased (Supplementary Fig. 6A). These findings suggested that the absence of EZH2 in DCs suppressed Th1 cells but promoted Treg differentiation during bacteria-induced liver injury. 


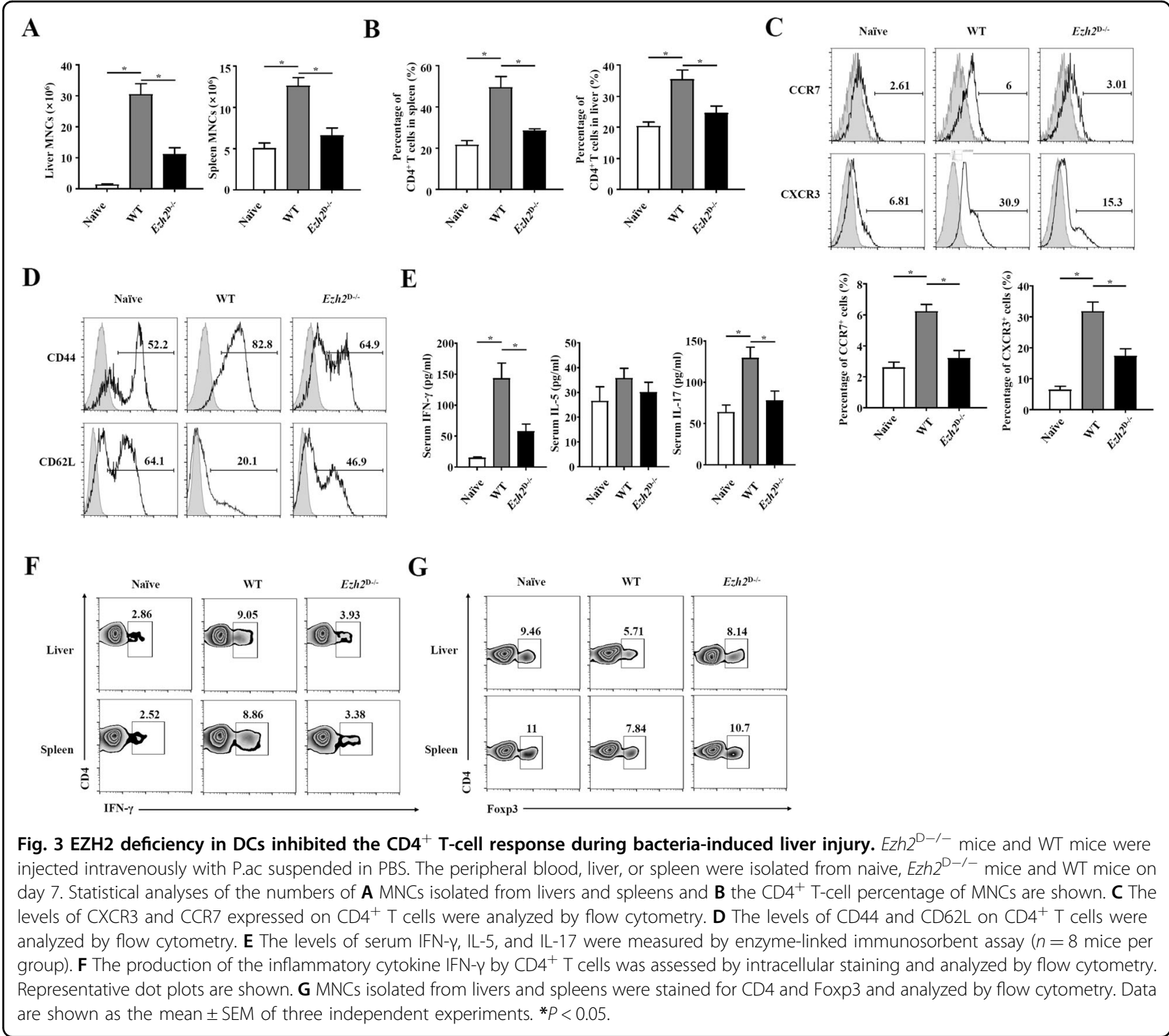

\section{Deficiency in EZH2 inhibited the maturation of DCs}

In our previous studies, it was shown that circulating $\mathrm{CD}_{11 \mathrm{c}^{+}} \mathrm{B}^{2} 20^{-} \mathrm{DC}$ precursors can be mobilized to the liver, where they differentiate into mature DCs later to function in immune responses ${ }^{13}$. Here, we determined whether EZH2 alters the characteristics of DC precursors. As shown, the percentage of DC precursors in the peripheral blood of mice increased after $P$. acnes priming; however, $E z h 2^{\mathrm{D}-1-}$ and WT mice showed no significant difference in the percentage of CD11c ${ }^{+} \mathrm{B} 220^{-}$cells (Fig. $4 \mathrm{~A}, \mathrm{~B})$, suggesting that EZH2 has no effect on DC precursor induction in the peripheral blood. In the liver injury model, the interactions between DCs and T cells are amplified by the continuous stimulation of liverinfiltrating $\mathrm{CD}^{+} \mathrm{T}$ cells by DCs, leading to subsequent liver injury ${ }^{35}$. Here, we investigated the characteristics of liver DCs. We isolated CD11 $\mathrm{c}^{+}$cells from the liver of $P$. acnes-induced mice and found that those from $E z h 2^{\mathrm{D}-1-}$ mice had lower expression of MHC class II (MHCII) and costimulatory molecules, including CD80 and CD86, than those from WT mice (Fig. 4C, D), indicating that deficiency in EZH2 inhibits the maturation of liver DCs. To further verify this hypothesis, we generated bone marrowderived DCs in vitro and activated them 6 days later with LPS. The results showed that EZH2 deficiency dramatically reduced the expression of CD80, CD86, and MHCII in CD11c ${ }^{+}$DCs (Fig. 4E, F). Furthermore, in a functional study, DCs generated from $E z h 2^{\mathrm{D}-/-}$ mice showed a lower capability to evoke an allogeneic mixed lymphocyte reaction, suppressing allogeneic $\mathrm{CD} 4^{+} \mathrm{T}$-cell proliferation in a dose-dependent manner, than DCs from WT mice (Fig. 4G). These findings demonstrated that the absence of EZH2 did not influence the induction of DC precursors but led to reduced DC maturation. 


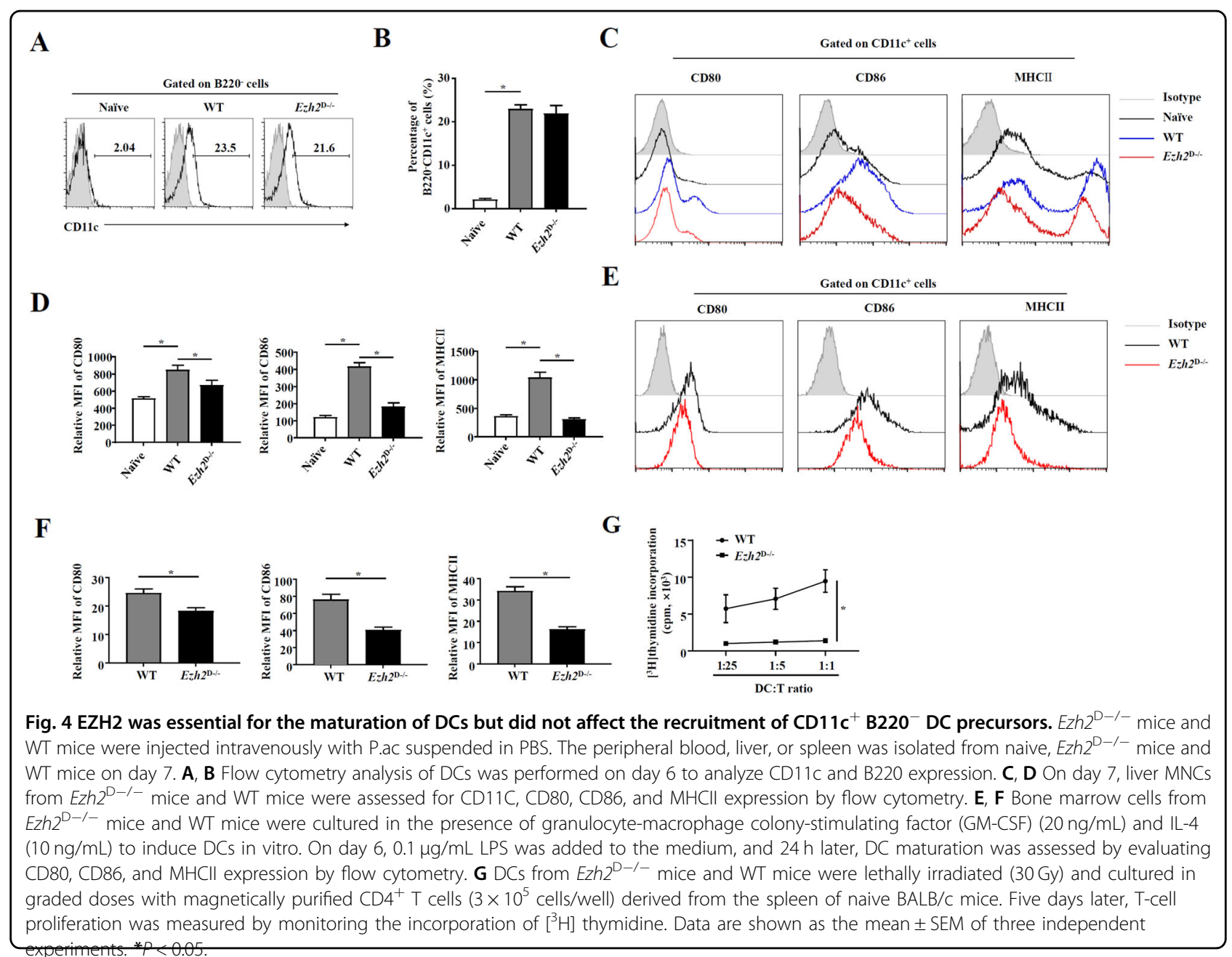

EZH2 repressed RUNX1 expression to regulate DC function

The transcription factor RUNX1 is essential for hematopoietic stem cell formation and bone marrow progenitor differentiation, such as DC lineage differentiation. RUNX1 has also been suggested to regulate DC functions ${ }^{36,37}$. As EZH2 was found to interact with RUNX1 in previous research ${ }^{38}$, we hypothesized that EZH2 regulates DC function by interacting with RUNX1. The expression of RUNX1 in DCs isolated from $E z h 2^{\mathrm{D}-1-}$ and WT mice was detected. We found that DC-specific deficiency in EZH2 upregulated the expression of RUNX1 (Fig. 5A, B) and repressed H3K27me3 levels in the RUNX1 promoter (Fig. 5C). To explore the function of RUNX1 and its interaction with EZH2, we treated bone marrow-derived DCs generated from $E z h 2^{\mathrm{D}-1-}$ and WT mice with the RUNX1 inhibitor Ro 5-3335 and found that Ro 5-3335 treatment notably upregulated the expression of CD80, CD86, and MHCII, which was downregulated in the context of EZH2 deficiency (Fig. 5D, E). In addition, in a functional study, suppression of RUNX1 in the context of EZH2 deficiency in DCs substantially decreased the capability to evoke an allogeneic mixed lymphocyte reaction (MLR) (Fig. 5F). To further explore effects on the chemotaxis and activation of pathogenic $\mathrm{CD} 4^{+} \mathrm{T}$ cells, we tested the expression levels of CCR7 and CXCR3 (related to chemotaxis) and those of CD44 and CD62L (related to activation). According to the results, which were similar to those of the functional study described above, suppression of RUNX1 in the context of EZH2 deficiency in DCs substantially reduced the capabilities to activate $\mathrm{CD}^{+}{ }^{+} \mathrm{T}$ cells and promote $\mathrm{CD} 4^{+} \mathrm{T}$-cell chemotaxis (Fig. 5G, H). The proportional changes in Th1 cells and Tregs were measured by detecting the intracellular cytokines IFN- $\gamma$ and Foxp3, which showed trends similar to those in the previously described assays (Fig. 5I). In addition, we examined the effects of Ro-3335 on T-cell survival and proliferation, and the results showed that there were no significant differences in the effects on $T$ cells, suggesting that RUNX1 inhibition does not have a direct effect on T-cell function (Supplementary Fig. 7A). These results suggest that RUNX1 is suppressed by EZH2 and that EZH2 functions through regulating RUNX1 in DCs. 


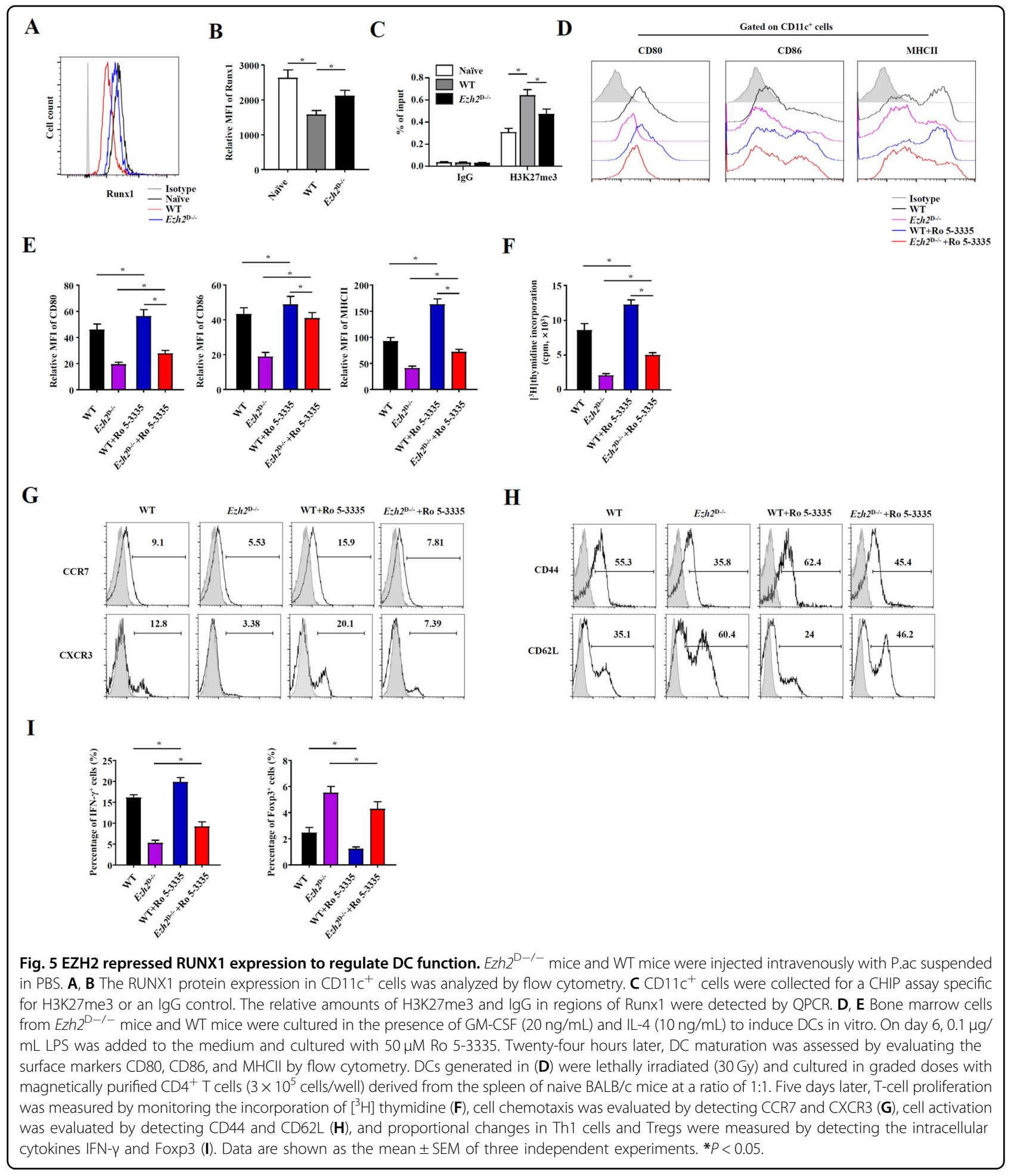

Inhibition of RUNX1 ameliorated the severity of bacteriainduced liver injury in Ezh2 ${ }^{\mathrm{D}-1-}$ mice

To further explore the function of RUNX1 and its interaction with $\mathrm{EZH} 2$ in liver injury, we treated $E z h 2^{\mathrm{D}-1-}$ and WT model mice with the RUNX1 inhibitor Ro 5-3335 to examine whether RUNX1 suppression can enhance FHF. As expected, we found that all of the $E z h 2^{\mathrm{D}-1-}$ and WT mice treated with Ro 5-3335 died relatively soon after LPS injection (Fig. 6A). We also detected ALT and AST levels and found that there were dramatic decreases in 


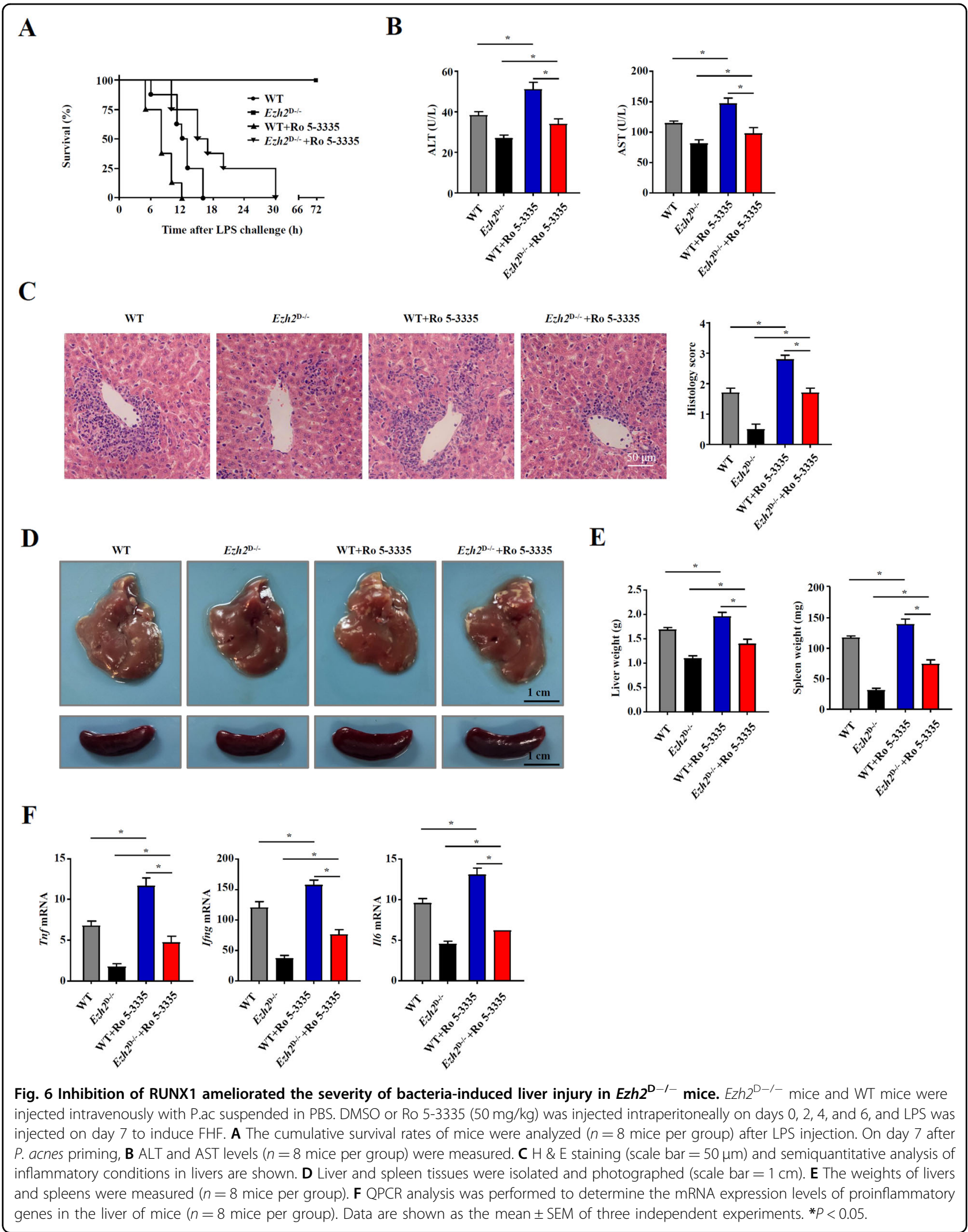


these levels in $E z h 2^{\mathrm{D}-1-}$ and WT mice treated with Ro 53335 (Fig. 6B). Histological analysis showed that larger nodules and more severe infiltration of lymphocytes were observed in liver tissue with Ro 5-3335 treatment (Fig. 6C). Furthermore, livers isolated from $E z h 2^{\mathrm{D}-1-}$ mice treated with Ro 5-3335 had more granulomas (Fig. $6 \mathrm{D})$. The weights of livers and spleens were also detected, and those from $E z h 2^{\mathrm{D}-1-}$ mice treated with Ro 5-3335 were heavier than those from mice only primed with $P$. acnes (Fig. 6E). Moreover, the mRNA expression of inflammatory cytokine genes, such as TNF- $\alpha$, IFN- $\gamma$, and IL-6, was elevated in the liver (Fig. 6F). Taken together, these findings suggest that EZH2 interacts with RUNX1 to regulate DC function during bacteria-induced liver injury and that suppression of RUNX1 enhances the severity of bacteria-induced liver injury.

\section{The EZH2 inhibitor GSK126 protected mice from bacteria- induced liver injury}

Recently, several highly selective small-molecule inhibitors targeting EZH2, such as GSK126, have been developed. To test the effect of an EZH2 inhibitor on liver injury, GSK126 was administered intraperitoneally to C57BL/6 mice on days $0,2,4$, and 6 after P. acnes priming. Then, LPS was injected on day 7 , and the survival rate of the mice was investigated. We found that all mice receiving GSK126 treatment survived for $72 \mathrm{~h}$, while the control mice died within $18 \mathrm{~h}$, suggesting that GSK126 treatment can dramatically promote survival (Fig. 7A). Then, we analyzed ALT and AST levels and found that they were significantly lower in GSK126-treated mice (Fig. 7B). Consistent with the improved survival rate of mice, histology related to liver injury and associated inflammatory factors was dramatically ameliorated in the GSK126-treated group (Fig. 7C-F). Moreover, the levels of the cytokines TNF- $\alpha$, IFN- $\gamma$, and IL- 6 in the serum were tested, and the results showed the same trends as the mRNA expression levels in the liver (Fig. 7G). These results suggested that treatment with the EZH2 inhibitor GSK126 successfully inhibited $P$. acnes-induced liver inflammation and ultimately prevented liver injury.

\section{Discussion}

This study aimed to investigate the role of EZH2 in the immune functions of DCs and its epigenetic mechanism in FHF. Here, we have shown that EZH2 deficiency established by gene knockout or inhibition of EZH2 with a small-molecule inhibitor impairs the immune responses of monocyte-derived and liver-resident DCs, which corresponds with the amelioration of bacteria-induced liver injury, increasing the survival rate of mice subjected to $P$. acnes plus LPS-induced FHF. Mechanistically, inhibition of EZH2 enhanced the expression of RUNX1 in DCs. This is the first study using EZH2 deficiency in conditional gene knockout mice to accurately explore the role of EZH2 in FHF.

Accumulating evidence suggests that EZH2 actively regulates inflammation. EZH2 is important in mediating the formation of H3K27me3 associated with gene transcription suppression ${ }^{39}$. It has been shown to exert essential roles in T-cell differentiation and polarization to promote the immune response in alloimmunity, act as a macrophage lineage-specific mediator of autoimmune inflammation and control immune responses in inflammatory bowel disease by affecting the development of MDSCs $^{24,27,40}$. P. acnes-induced liver injury is reported to be attributed to Th1 cell-mediated inflammatory responses. In the present study, EZH2 and H3K27me3 levels were upregulated as liver injury progressed, suggesting that they are involved in liver failure by regulating immune responses. Epigenetic regulation has emerged as one of the key mechanisms regulating DC development and function. A histone deubiquitinase, Mysm1, has been reported to govern DC differentiation by modulating histone modifications and mediating recruitment of the transcription factor PU. $1^{18}$. In the current study, we observed that the expression of EZH2 and H3K27me3 increased in DCs during liver injury. This experimental evidence raises the possibility that EZH2 is involved in liver failure by regulating the development and function of DCs.

We demonstrated that genetic deletion of EZH2 in DCs or inhibition of its enzymatic activity substantially ameliorated liver injury by inhibiting the immune responses evoked by DCs. A recent study showed that EZH2 promotes the extravasation and motility of DCs and neutrophils under inflammatory conditions ${ }^{30}$. Moreover, EZH2 regulates Langerhans cell transmigration, and EZH2-deficient dermal DCs exhibit increased tolerogenicity $^{41}$. In this study, the number of $\mathrm{CD} 11 \mathrm{c}^{+} \mathrm{B} 220^{-}$ DCs in the liver showed no significant difference between $E z h 2^{\mathrm{D}-1-}$ mice and control mice, indicating that EZH2 deficiency does not affect the recruitment of DCs to the liver. The ability of DCs to stimulate $\mathrm{T}$ cells is related to DC maturation. In this study, EZH2 deficiency significantly reduced the expression of CD80, CD86, and MHCII in liver DCs and monocyte-derived DCs, and these DC populations showed reduced potency in stimulating $\mathrm{CD} 4^{+} \mathrm{T}$-cell proliferation. These findings suggest that EZH2 deficiency suppresses the phenotypic and functional activation of DCs. Although recent research provides experimental evidence indicating that the phenotypic and functional activation of moDCs was significantly suppressed by EZH2 inhibition with the inhibitor 3 Deazaneplanocin A (DZNep) ${ }^{32}$, we directly confirmed the effect of EZH2 on DCs in $E z h 2^{\mathrm{D}-1-}$ mice. According to the results of DC subset analysis during FHF, EZH2 expression correlated with the generation and 


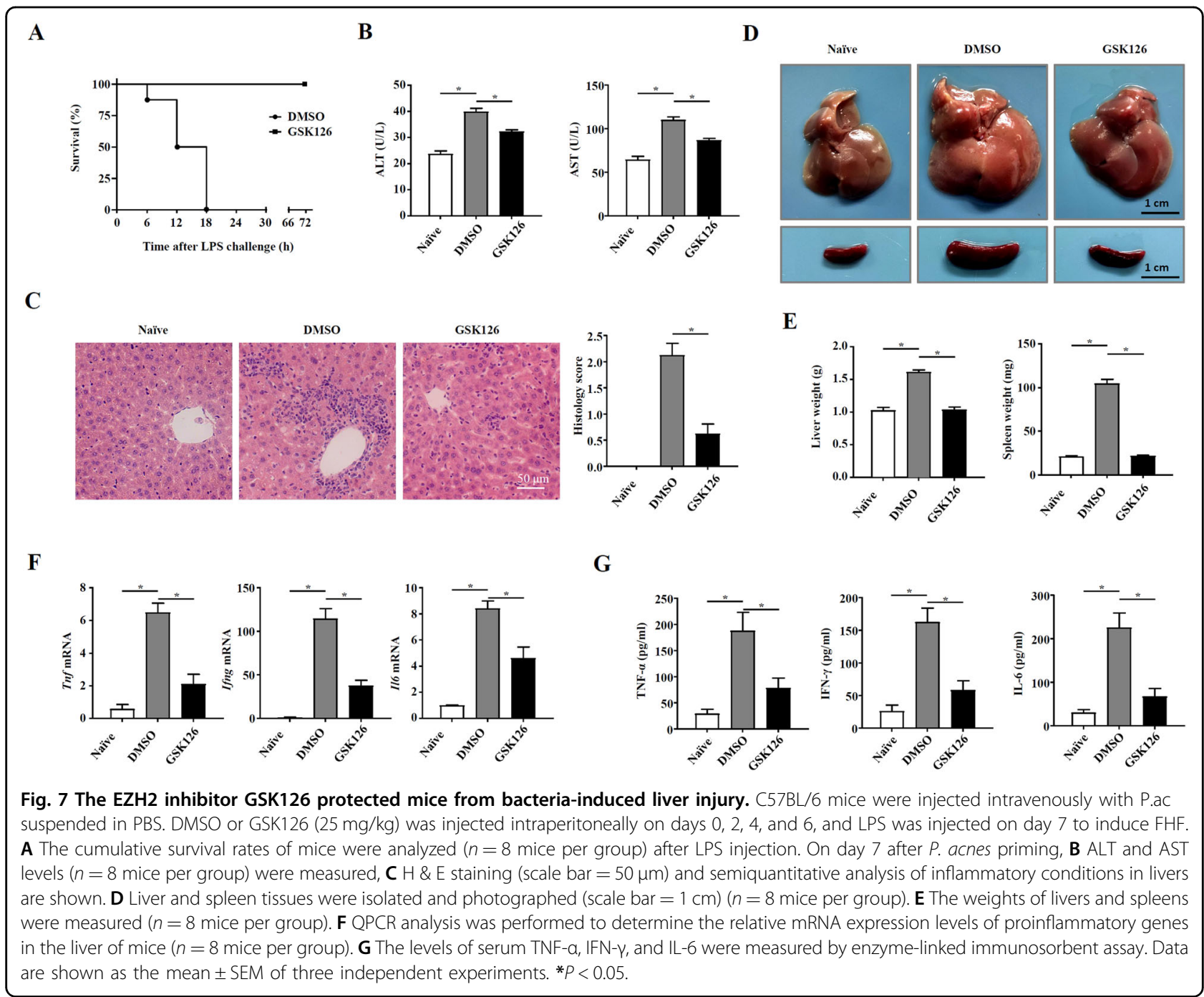

distribution of DC subsets in this disease, especially DCs from the spleen, a lymphoid organ. The underlying mechanisms will be further explored in prospective studies.

EZH2 functions depend on interactions with proteins, such as AKT, AP-1, and $\mathrm{CDK}^{42-44}$. We previously found that EZH2 could regulate the DNA damage-responsive protein $\mathrm{Ku}^{4} 0^{45}$ and identified the EZH2-Hsp90 interaction as an essential mechanism underlying $\mathrm{T}$-cell responses in graft-versus-host disease ${ }^{46}$. The transcription factor RUNX1 is a member of the Runt-related transcription factor family of genes encoding the DNAbinding $\alpha$-chain partners of the heterodimeric CBF complex $^{36,47}$. It has been demonstrated that RUNX1 can mediate bone morphogenetic protein signaling in human DC maturation ${ }^{48}$ and is required for the development of $\mathrm{Flt}^{+}{ }^{+}$DC progenitors and all mature DC lineages ${ }^{37}$. In androgen-dependent prostate cancer, the RUNX promoter is bound by EZH2 and negatively regulated by
H3K27me $3^{38}$. The interaction between EZH2 and RUNX1 was proven in our system. We observed that EZH2 deficiency enhanced the expression of RUNX1. EZH2-mediated regulation of immune responses induced by RUNX1 in DCs was demonstrated by inhibition of RUNX1 with its inhibitor Ro 5-3335 in the context of EZH2 deficiency both in vivo and in vitro. Inhibition of RUNX1 restored LPS-induced activation and DC-T cell responses in EZH2-deficient DCs, suggesting that EZH2 regulates the activation and immune functions dependent on RUNX1. However, according to the results, the effect of EZH2 deficiency in DCs was not linked to only RUNX1 expression. Other mechanisms, in addition to the mechanism involving RUNX1, may be involved. For example, the tumor necrosis factor (TNF) superfamily is a large family of molecules expressed by various immune cells and induced during the activation of the immune system. TNF superfamily members can influence DC biology, promoting homeostasis, maximizing $\mathrm{T}$ cell-DC 
encounters, and enhancing $\mathrm{T}$-cell proliferation and cytokine secretion in the immune response. It was reported that the TNF superfamily ligands $\mathrm{TNF}^{49}$ and CD40 ligand ${ }^{50}$ play key roles in promoting the proliferation of bone marrow-derived progenitor cells and their differentiation into DCs. In previous research, it was shown that EZH2-catalyzed H3K27 trimethylation plays a key role in acute-on-chronic liver failure via a TNF-mediated pathway ${ }^{29}$. The results suggested that EZH2/H3K27me3 promoted the progression of liver failure and that TNF, along with downstream NF-kB and Akt signaling pathways, was manipulated by epigenetic modification with H3K27me3. Therefore, the effect of EZH2 deficiency on DCs may be correlated with the regulation of the TNF pathway in addition to the regulation of RUNX1 expression. It was also verified in vivo that Ro 5-3335 treatment promoted the progression of FHF in $E z h 2^{\mathrm{D}-1-}$ mice. However, whether the RUNX1 promoter is associated with EZH2, the regions occupied by EZH2 and the binding sites for EZH2 in the RUNX1 gene remain to be further confirmed by CHIP assays in the future.

Impressive studies have demonstrated the therapeutic potential of EZH2 inhibition, and numerous highly selective small-molecule inhibitors targeting EZH2 have been developed, some of which have entered clinical development ${ }^{51,52}$. With the aim of exploring the methyltransferase activity of EZH2 and histone modification, we selected GSK126 based on its high selectivity in inhibiting methyltransferase by competing with $S$-denosylmethionine without degrading $\mathrm{EZH} 2^{53}$. GSK126 has been shown to ameliorate disease severity in acute-on-chronic liver failure by modulating TNF and overall inflammation $^{29}$. The effect of GSK126 was also confirmed in the current study, suggesting its potential clinical application in FHF.

Overall, our study investigated the roles of EZH2 in the immune functions of DCs and the epigenetic mechanisms of EZH2 in FHF, suggesting that EZH2 promotes the development of liver failure in FHF, which was verified with $E z h 2^{\mathrm{D}-1-}$ mice and ameliorated using the inhibitor GSK126, via modulation of immune responses. Moreover, inhibition of EZH2 enhanced the expression of RUNX1, which functions as a downstream effector of EZH2 in DCs. This is the first study to provide direct experimental evidence proving the significant role of DC EZH2 in FHF, supporting the potential clinical applicability of pharmacological targeting of epigenetic modifications for the treatment of FHF.

\section{Acknowledgements}

This work was supported by National Natural Science Foundation of China (81700656, 81670540, 82000567, 81900565, 81873447, 82000553) and The Program of Science and Technology Commission of Shanghai Municipality (19ZR1430900).

\section{Author details}

${ }^{1}$ Shanghai Institute of Immunology, Shanghai Jiao Tong University School of Medicine, Shanghai, China. ${ }^{2}$ CAS Key Laboratory of Tissue Microenvironment and Tumor, Shanghai Institute of Nutrition and Health, Chinese Academy of Sciences, Shanghai, China. ${ }^{3}$ Department of Stomatology, Renji Hospital, Shanghai Jiao Tong University School of Medicine, Shanghai, China. ${ }^{4}$ Institutes for Translational Medicine, Soochow University, Suzhou, China. ${ }^{5}$ Department of Oncology, Xinhua Hospital, Shanghai Jiao Tong University School of Medicine, Shanghai, China

\section{Conflict of interest}

The authors declare that they have no conflict of interest.

\section{Publisher's note}

Springer Nature remains neutral with regard to jurisdictional claims in published maps and institutional affiliations.

Supplementary Information accompanies this paper at (https://doi.org/ 10.1038/s41419-020-03219-w).

Received: 23 July 2020 Revised: 1 November 2020 Accepted: 3 November 2020

Published online: 01 December 2020

\section{References}

1. Hoofnagle, J. H. et al. Fulminant hepatic failure: summary of a workshop. Hepatology 21, 240-52 (1995).

2. Sass, D. A. \& Shakil, A. O. Fulminant hepatic failure. Liver Transpl. 11, 594-605 (2005).

3. Stravitz, R. T. \& Lee, W. M. Acute liver failure. Lancet 394, 869-881 (2019).

4. Yoneyama, $\mathrm{H}$. et al. Pivotal role of TARC, a CC chemokine, in bacteria-induced fulminant hepatic failure in mice. J. Clin. Invest. 102, 1933-1941 (1998).

5. Zhang, Y. et al. Mesenchymal stem cells alleviate bacteria-induced liver injury in mice by inducing regulatory dendritic cells. Hepatology 59, 671-682 (2014).

6. Xiao, Y. et al. 18Beta-glycyrrhetinic acid ameliorates acute Propionibacterium acnes-induced liver injury through inhibition of macrophage inflammatory protein-1alpha. J. Biol. Chem. 285, 1128-1137 (2010).

7. Nakayama, Y. et al. CTLA-4lg suppresses liver injury by inhibiting acquired immune responses in a mouse model of fulminant hepatitis. Hepatology $\mathbf{4 2}$, 915-924 (2005).

8. Sabado, R. L., Balan, S. \& Bhardwaj, N. Dendritic cell-based immunotherapy. Cell Res. 27, 74-95 (2017).

9. Ananiev, J. et al. Macrophages and dendritic cells in the development of liver injury leading to liver failure. J. Biol. Regul. Homeost. Agents 28, 789-794 (2014).

10. Bamboat, Z. M. et al. Conventional DCs reduce liver ischemia/reperfusion injury in mice via IL-10 secretion. J. Clin. Invest. 120, 559-569 (2010).

11. Yoshida, O. et al. CD39 expression by hepatic myeloid dendritic cells attenuates inflammation in liver transplant ischemia-reperfusion injury in mice. Hepatology 58, 2163-2175 (2013).

12. Koda, Y. et al. Plasmacytoid dendritic cells protect against immune-mediated acute liver injury via IL-35. J. Clin. Invest. 129, 3201-3213 (2019).

13. Yoneyama, $\mathrm{H}$. et al. Regulation by chemokines of circulating dendritic cell precursors, and the formation of portal tract-associated lymphoid tissue, in a granulomatous liver disease. J. Exp. Med. 193, 35-49 (2001).

14. Gu, Q. et al. Heme oxygenase-1 alleviates mouse hepatic failure through suppression of adaptive immune responses. J. Pharm. Exp. Ther. 340, 2-10 (2012).

15. Khanam, A. et al. Altered frequencies of dendritic cells and IFN-gammasecreting T cells with granulocyte colony-stimulating factor (G-CSF) therapy in acute-on- chronic liver failure. Liver Int. 34, 505-513 (2014).

16. Connolly, M. K. et al. Dendritic cell depletion exacerbates acetaminophen hepatotoxicity. Hepatology 54, 959-968 (2011).

17. Zhang, Y. et al. Mobilization of dendritic cell precursors into the circulation by administration of MIP-1alpha in mice. J. Natl Cancer Inst. 96, 201-209 (2004).

18. Won, $\mathrm{H}$. et al. Epigenetic control of dendritic cell development and fate determination of common myeloid progenitor by Mysm1. Blood 124, 2647-2656 (2014)

19. Tian, Y., Meng, L. \& Zhang, Y. Epigenetic regulation of dendritic cell development and function. Cancer J. 23, 302-307 (2017). 
20. Cao, R. et al. Role of histone $\mathrm{H} 3$ lysine 27 methylation in Polycomb-group silencing. Science 298, 1039-1043 (2002).

21. Margueron, R. \& Reinberg, D. The Polycomb complex PRC2 and its mark in life. Nature 469, 343-349 (2011).

22. Su, I. H. et al. Ezh2 controls B cell development through histone H3 methylation and lgh rearrangement. Nat. Immunol. 4, 124-131 (2003)

23. Guo, M. et al. EZH2 represses the B cell transcriptional program and regulates antibody-secreting cell metabolism and antibody production. J. Immunol. 200, 1039-1052 (2018)

24. Tumes, D. J. et al. The polycomb protein Ezh2 regulates differentiation and plasticity of CD4(+) T helper type 1 and type 2 cells. Immunity 39, 819-832 (2013).

25. Zhang, Y. et al. The polycomb repressive complex 2 governs life and death of peripheral T cells. Blood 124, 737-749 (2014).

26. He, S. et al. Histone methyltransferase and histone methylation in inflammatory T-cell responses. Immunotherapy 5, 989-1004 (2013).

27. Zhang, $X$. et al. Macrophage/microglial Ezh2 facilitates autoimmune inflammation through inhibition of Socs3. J. Exp. Med. 215, 1365-1382 (2018).

28. Mann, J. et al. MeCP2 controls an epigenetic pathway that promotes myofibroblast transdifferentiation and fibrosis. Gastroenterology 138, 705-714 (2010).

29. Zhou, T. et al. Enhancer of zeste homolog 2-catalysed H3K27 trimethylation plays a key role in acute-on-chronic liver failure via TNF-mediated pathway. Cell Death Dis. 9, 590 (2018).

30. Gunawan, M. et al. The methyltransferase Ezh2 controls cell adhesion and migration through direct methylation of the extranuclear regulatory protein talin. Nat. Immunol. 16, 505-516 (2015).

31. Tian, $X$. et al. Expression of enhancer of zeste homolog $2(E Z H 2)$ protein in histiocytic and dendritic cell neoplasms with evidence for p-ERK1/2-related, but not MYC- or p-STAT3-related cell signaling. Mod. Pathol. 31, 553-561 (2018).

32. Li, H. et al. Epigenetic modification of enhancer of zeste homolog 2 modulates the activation of dendritic cells in allergen immunotherapy. Int. Arch. Allergy Immunol. 180, 120-127 (2019).

33. Xiao, Y. et al. Genetic ablation of steroid receptor coactivator-3 promotes PPAR-beta-mediated alternative activation of microglia in experimental autoimmune encephalomyelitis. Glia 58, 932-942 (2010).

34. $\mathrm{Xu}$, J. et al. Tpl2 protects against fulminant hepatitis through mobilization of myeloid-derived suppressor cells. Front Immunol. 10, 1980 (2019).

35. Yoneyama, $\mathrm{H}$. et al. Pivotal role of dendritic cell-derived CXCL10 in the retention of $\mathrm{T}$ helper cell 1 lymphocytes in secondary lymph nodes. J. Exp. Med. 195, 1257-1266 (2002).

36. Chen, M. J. et al. Runx1 is required for the endothelial to haematopoietic cell transition but not thereafter. Nature 457, 887-891 (2009).
37. Satpathy, A. T. et al. Runx1 and Cbfbeta regulate the development of Flt3+ dendritic cell progenitors and restrict myeloproliferative disorder. Blood 123, 2968-2977 (2014).

38. Takayama, K. et al. RUNX1, an androgen- and EZH2-regulated gene, has differential roles in AR-dependent and -independent prostate cancer. Oncotarget 6, 2263-2276 (2015).

39. Allis, C. D. \& Jenuwein, T. The molecular hallmarks of epigenetic control. Nat. Rev. Genet. 17, 487-500 (2016).

40. Zhou, J. et al. Targeting EZH2 histone methyltransferase activity alleviates experimental intestinal inflammation. Nat. Commun. 10, 2427 (2019).

41. Loh, J. T. et al. Ezh2 controls skin tolerance through distinct mechanisms in different subsets of skin dendritic cells. iscience 10, 23-39 (2018).

42. De Santa, F. et al. The histone H3 lysine-27 demethylase Jmjd3 links inflammation to inhibition of polycomb-mediated gene silencing. Cell 130, 1083-1094 (2007).

43. Cha, T. L. et al. Akt-mediated phosphorylation of EZH2 suppresses methylation of lysine 27 in histone H3. Science 310, 306-310 (2005).

44. Wei, Y. et al. CDK1-dependent phosphorylation of EZH2 suppresses methylation of $\mathrm{H} 3 \mathrm{~K} 27$ and promotes osteogenic differentiation of human mesenchymal stem cells. Nat. Cell Biol. 13, 87-94 (2011).

45. Wang, Y. et al. DNA-PK-mediated phosphorylation of EZH2 regulates the DNA damage-induced apoptosis to maintain T-cell genomic integrity. Cell Death Dis. 7, e2316 (2016).

46. Huang, Q. et al. Hsp90 inhibition destabilizes Ezh2 protein in alloreactive $\mathrm{T}$ cells and reduces graft-versus-host disease in mice. Blood 129, 2737-2748 (2017).

47. Sood, R., Kamikubo, Y. \& Liu, P. Role of RUNX1 in hematological malignancies. Blood 129, 2070-2082 (2017).

48. Martinez, V. G. et al. The canonical BMP signaling pathway is involved in human monocyte-derived dendritic cell maturation. Immunol. Cell Biol. 89 610-618 (2011).

49. Caux, C. et al. CD34+ hematopoietic progenitors from human cord blood differentiate along two independent dendritic cell pathways in response to GM-CSF+TNF alpha. Adv. Exp. Med. Biol. 417, 21-25 (1997).

50. Flores-Romo, L. et al. CD40 ligation on human cord blood CD34+ hematopoietic progenitors induces their proliferation and differentiation into functional dendritic cells. J. Exp. Med. 185, 341-349 (1997).

51. Kim, K. H. \& Roberts, C. W. Targeting EZH2 in cancer. Nat. Med. 22, 128-34 (2016).

52. Stephenson, R. \& Singh, A. Drug discovery and therapeutic delivery for the treatment of B and T cell tumors. Adv. Drug Deliv. Rev. 114, 285-300 (2017).

53. McCabe, M. T. et al. EZH2 inhibition as a therapeutic strategy for lymphoma with EZH2-activating mutations. Nature 492, 108-112 (2012). 